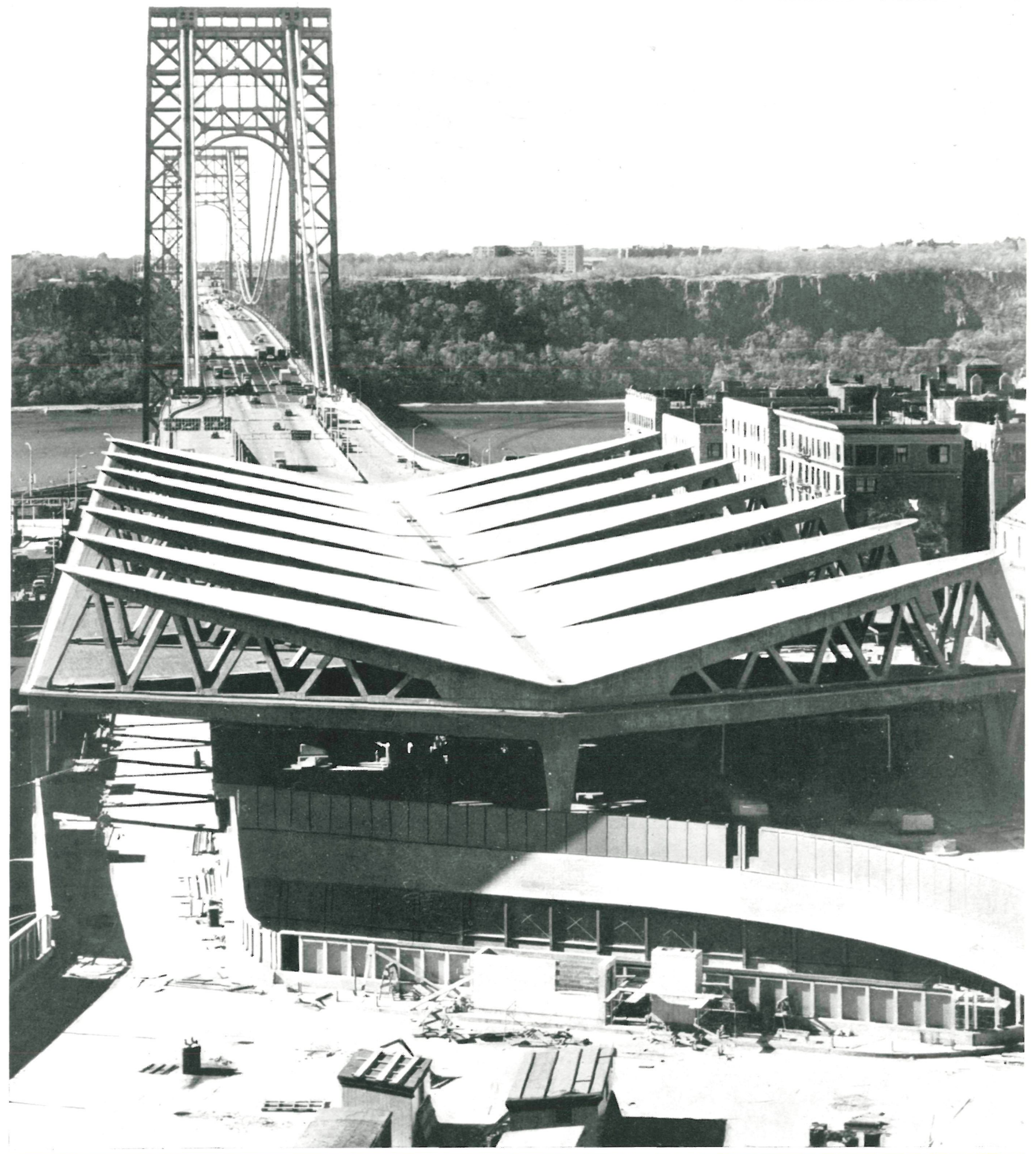

\title{
nueva estación de autobuses en Nueva York
}

PIER LUIGI NERVI, ingeniero

sำ 10 psis

$144-19$

La estación de autobuses, recientemente construida en las proximidades del puente Washington (Nueva York), es un terminal de la línea Nueva York-Nueva Jersey. Su acceso se realiza mediante rampas que terminan en una plataforma con diez andenes, en los que pueden estacionarse 36 autobuses simultáneamente. Tiene capacidad para unos 2.000 estacionamientos diarios.

Dadas las condiciones particulares del edificio, la plataforma superior está completamente abierta para facilitar la ventilación. La estructura, de hormigón armado, tiene tres plantas: una para los servicios mecánicos y acceso, otra que aloja las taquillas, escaleras mecánicas y servicios generales, y otra en la que están los diez andenes de estacionamiento de autobuses. 


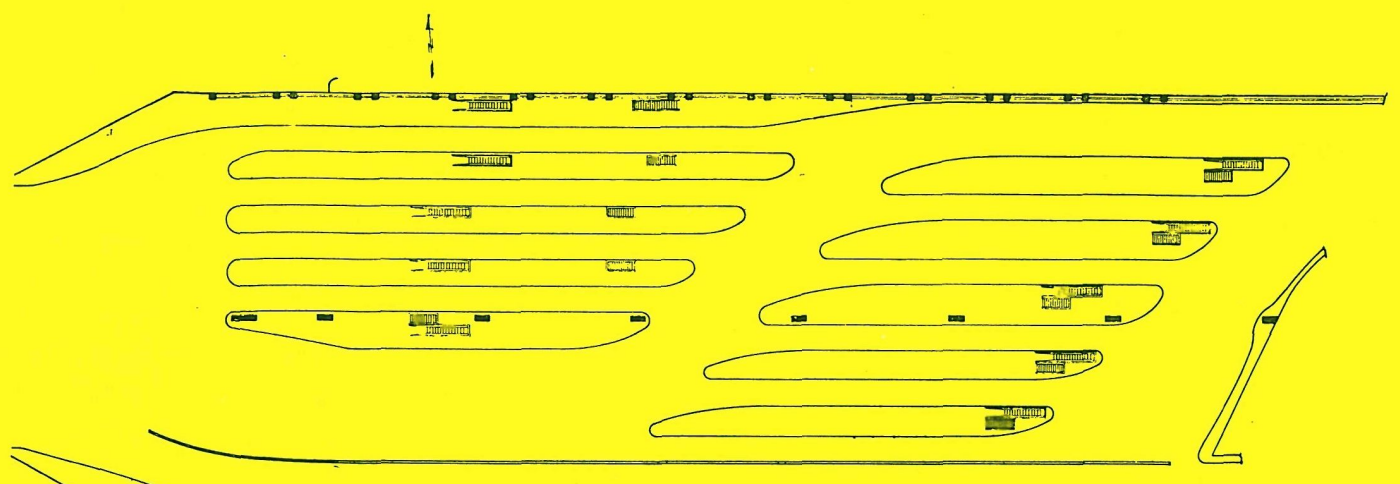

A Planta de un elemento de cubierta

B Sección A-A

C Sección B-B

D Planta baja

E Planta primera

F Planta superior
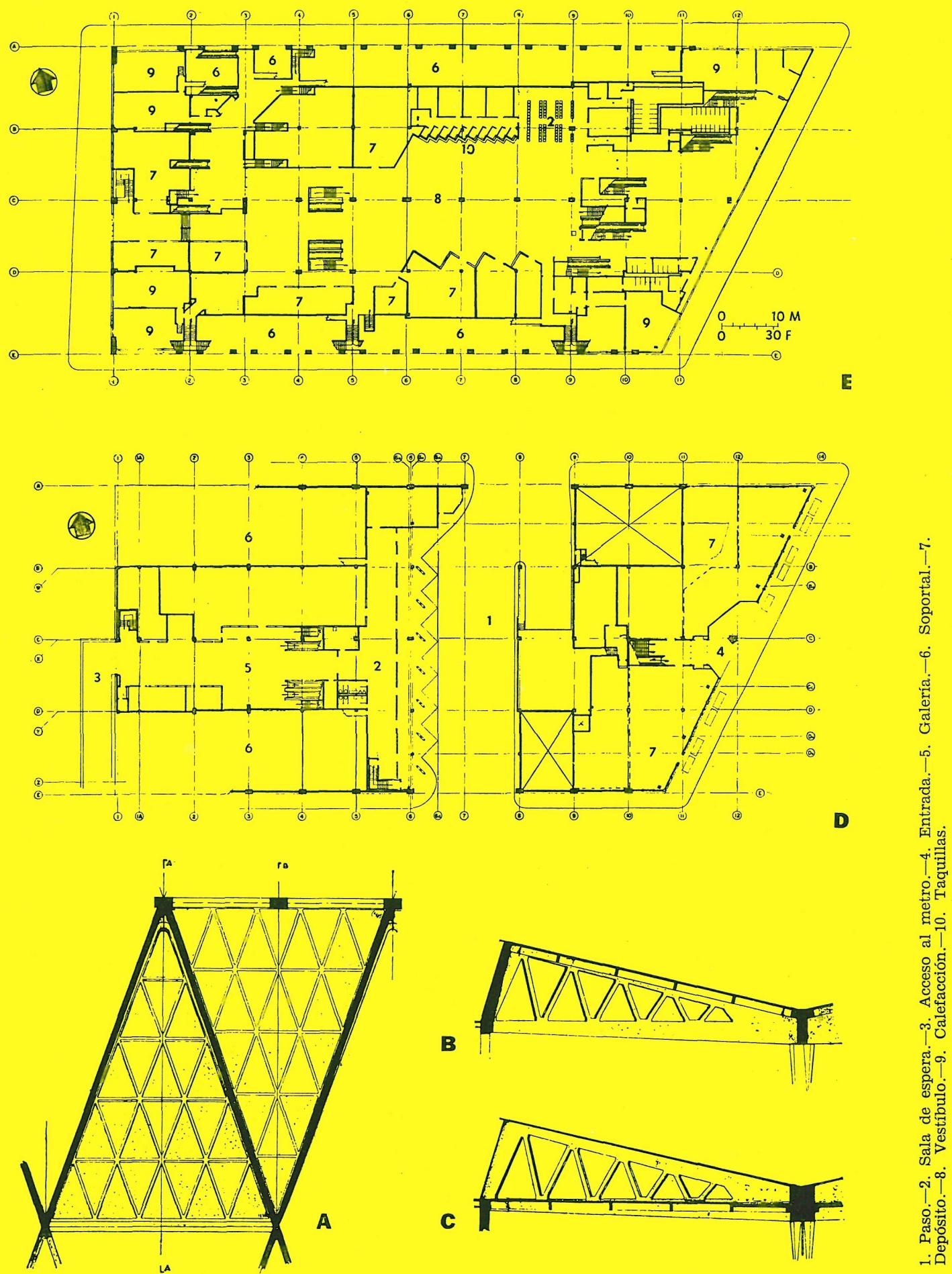


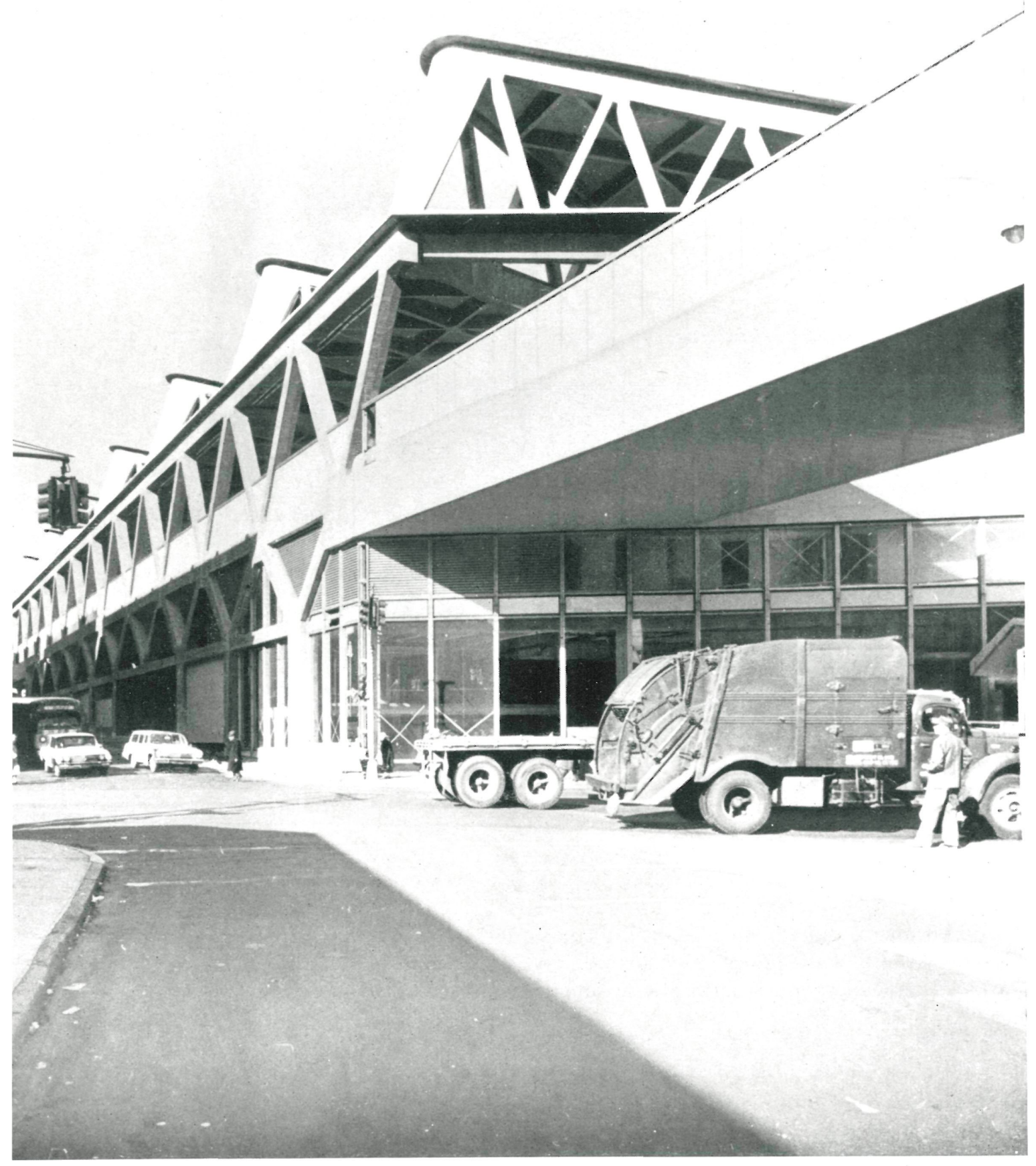

Paso inferior.

La construcción presenta una red de triangulaciones o celosías aparentes, tanto desde el exterior como desde el interior.

La cubierta, de forma particular, se compone de 14 elementos tetraédricos cuyas caras laterales son celosías trianguladas. Estos elementos se apoyan, en la parte central del edificio, sobre una fila de montantes articulados en sus bases y, al exterior, sobre los muros de las fachadas.

Aunque todos los elementos que forman la estructura son muy robustos, el conjunto resulta de una gran esbeltez y se ha hormigonado totalmente en obra, dejando el hormigón visto.

La estación de autobuses construida en las inmediaciones del puente Washington, por cuenta de la Port of New York Authority, tiene capacidad para 18 millones de pasajeros anuales, con una media de 2.000 autobuses y 50.000 pasajeros diarios. La planta es un rectángulo de $122 \times 56,6 \mathrm{~m}$, lo que permite un estacionamiento simultáneo de 200 autobuses. 


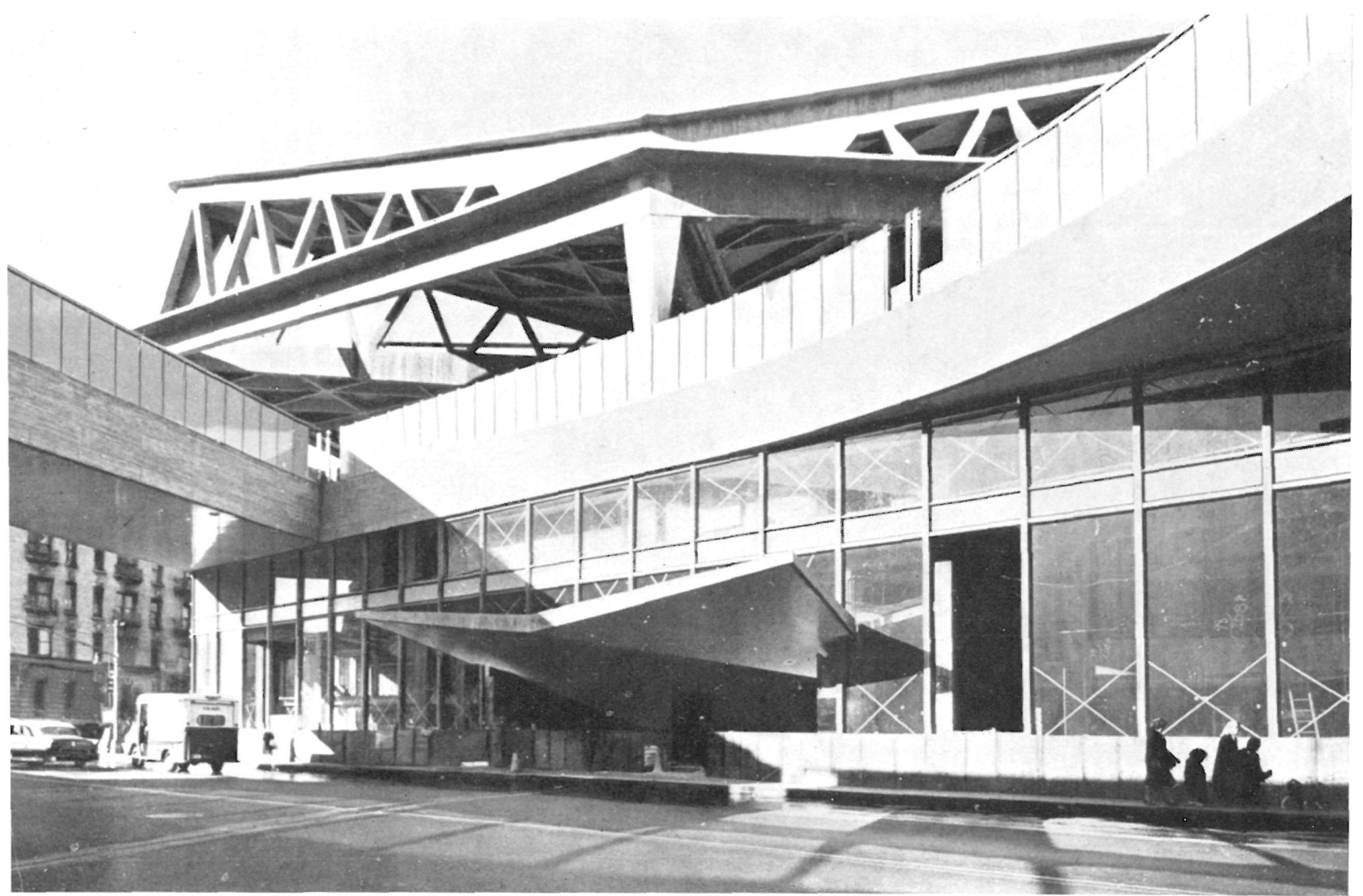

Entrada del personal.

La estructura es de hormigón armado sin tratamiento posterior, dejando el material tal como salió de los encofrados. Para que el aspecto de los paramentos fuera agradable a la vista, los áridos empleados en el hormigón se sometieron a un estudio previo y ensayos pertinentes.

El edificio tiene tres plantas y su acceso inferior puede ser utilizado por el personal procedente del «metro» o directamente desde la calle. En este nivel se hallan los talleres y depósitos. La planta intermedia está destinada a los pasajeros y cuenta con un gran vestíbulo, espera, tiendas escaparates, bar, restaurante, taquillas y servicios de información. El acceso de esta planta a la superior o estación propiamente dicha, se realiza por medio de escaleras mecánicas que desembocan en diez andenes o plataformas en las que se pueden estacionar hasta 36 autobuses.

Por razones imperativas, la planta superior no se ha cerrado y todos sus huecos permiten una ventilación óptima. Las instalaciones mecánicas pesadas se hallan en el sótano.

La estructura, hormigonada en obra, es del tipo triangulado, rígida, apreciable tanto en las fachadas como desde el interior. Dicha triangulación constituye los muros y soportes de los forjados de la segunda y tercera planta, siendo esta última la de mayor importancia en cuanto a sobrecargas se refiere.

La cubierta de la estación presenta una forma particular, componiéndose de dos series, de siete elementos cada una, constituyendo los faldones que vierten aguas hacia la parte central. En planta estos elementos tienen forma triangular, y considerados aisladamente, su figura geométrica más similar sería la de un tetraedro con caras laterales constituyendo celosía abierta triangulada. 
Los elementos tetraédricos de cubierta se apoyan, en la parte central de la cubierta, sobre una fila de montantes robustos, de hormigón, articulados en sư base, y en las extremidades, al exterior, sobre los muros o celosías trianguladas de las fachadas.

A pesar del gran volumen de los elementos de cubierta, el conjunto resulta de una gran esbeltez. Las losas triangulares de cerramiento superior son nervadas, siendo el triángulo o la $\mathrm{V}$ los temas básicos en todas las partes del edificio y en su conjunto.

El interior-principalmente el gran vestíbulo - ha sido decorado con una combinación de blanco y azul; revestimientos verticales de mosaico vítreo, solados de terrazo pulimentado e iluminación artificial con tubos de luz fluorescente empotrados en un techo acústico de aluminio.

Esta obra es la primera del país en que se deja el hormigón basto, sin tratamiento alguno, como queda al desencofrar, ya que las tendencias actuales se inclinan decididamente hacia esta técnica decorativa.

El proyectista había previsto la prefabricación para la mayor parte de los elementos; pero por razones de economía se introdujo el hormigonado en obra, después de un detallado estudio de las circunstancias que aconsejaban este procedimiento.

La estructura ha despertado general interés dentro del campo de la construcción, con su valoración del hormigón visto como medio decorativo.

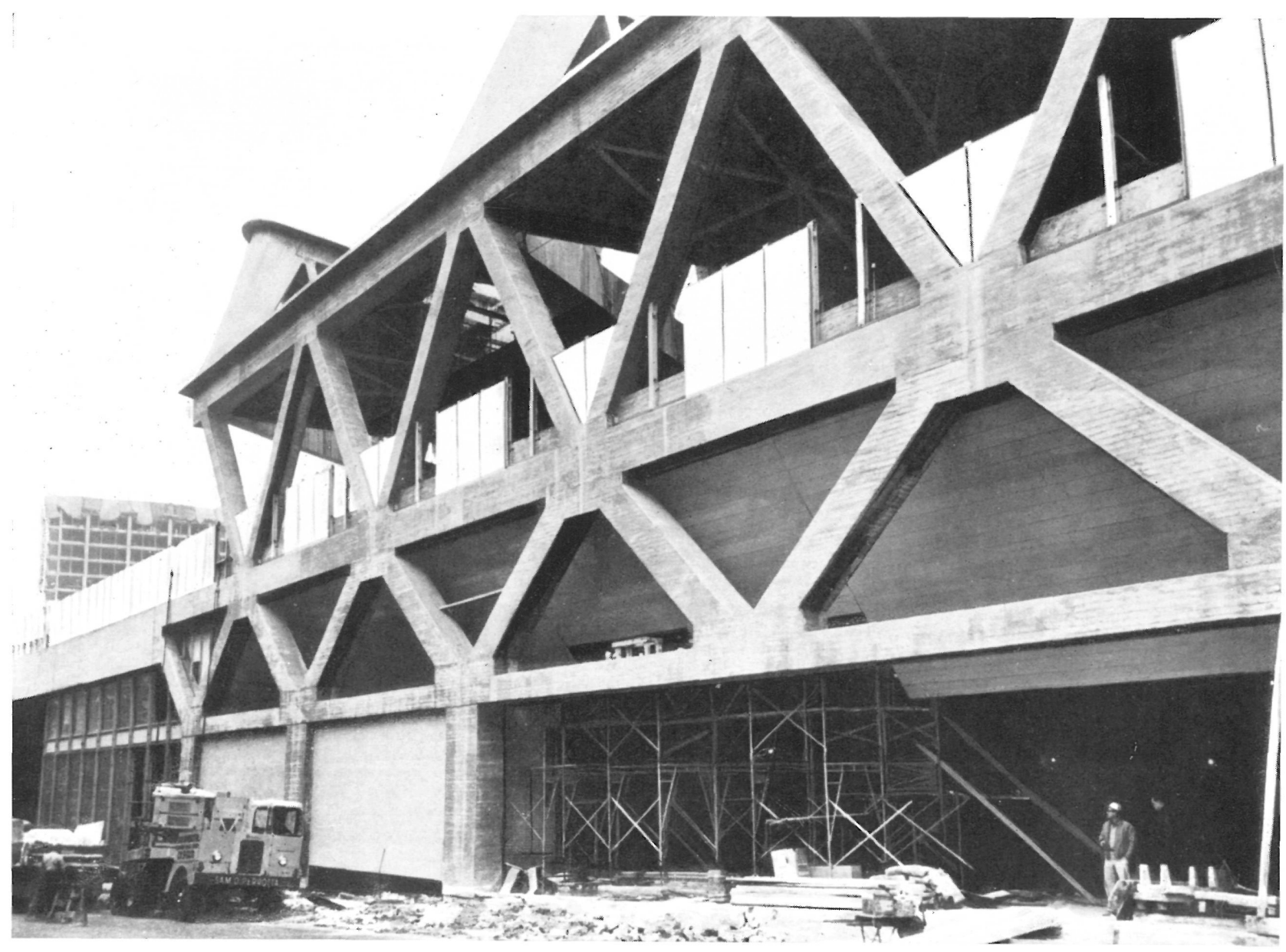




\section{Mouvelle statrion d"antobus di New York}

Pier Luigi Nervi, ingénieur

La station d'autobus, récemment construite à proximité du pont Washington (New York), est un terminus de la ligne New YorkNew Jersey. Son accès se réalise à l'aide de rampes qui se terminent sur une plate-forme de dix quais permettant le station. nement simultané de 36 autobus. La capacité est de 2.000 stationnements environ par jour.

Etant donné les conditions particulières de l'édifice, la plate-forme supérieure est complètement ouverte pour faciliter la ventilation. La structure, en béton armé, a trois étages: le premier pour les services mécaniques et accès, le deuxième pour les guichets, escaLa structure, en béton armé, a trois étages: le premier pour les services mécaniques et accès, le deux
liers mécaniques et services généraux, et le troisième pour les 10 quais de stationnement des autobus.

La construction présente un réseau de triangulations ou poutres en treillis apparentes, tant de l'extérieur que de l'intérieur.

La couverture, d'une forme particulière, se compose de 14 éléments tétraédriques dont les surfaces latérales sont formées par La couverture, d'une forme particulière, se compose de 14 éléments tétraédriques dont les surfaces latérales sont formées par bases et, à l'extérieur. Cur les murs de faude.

Bien que tous les éléments formant la structure soient très robustes, cet ensemble, d'un grand élancement, a été bétonné totalement à pied d'oeuvre et le béton a été laissé nu.

\section{New Buss Strotion in New Mork}

Pier Luigi Nervi, engineer.

The bus station, recently built near Washington Bridge, New York, is a terminal of the New York to New Jersey line. It is reached by ramps which lead to its 10 platforms, where 36 buses can be parked simultaneously. Up to 2000 buses can use the station in one day.

Bcause of the special circumstances of the building, the top platform is entirely open, to facilitate ventilation. The structure of reinforced concrete supports three floor levels. One is taken up with workshop servicing and entrances, the second has ticket offices, escalators and services, and the third constitutes the station proper, with the ten bus platforms.

The structure involves open frameworks, which are visible both from inside and outside.

The special type roof consists of 14 tetrahedral units, whose lateral faces are open triangulations. These rest on a series of columns, hinged at the bottom, placed along the centre of the building, and on the external walls.

Although all structural elements are very strong, the total design looks slender and attractive. Concreting was done at the site, and the concreted surfaces have been left uncovered.

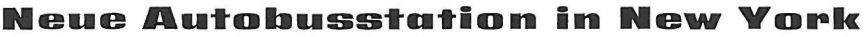

Pier Luigi Nervi, Ingenieur

Die kürzlich gebaute Autobusstation in der Nähe der Washingtonbrücke (New York), ist die Endstation der Linie New York-New Jersey. Die Auffahrt geschieht ïber eine Reihe von Rampen die in einer Plattform mit 10 Fussteigen enden, auf der 36 Busse gleichzeitig parken können. Die ganze Station hat eine Kapazität von 2.000 Bussen täglich.

Auf Grund der Eigenart dieses Gebäudes ist die obere Plattform völlig offen, um die Lüftung zu erleichtern. Die Stahlbetonstruktur besteht aus drei Stockwerken: eines für Reparaturwerkstätte und Zufahrten; ein weiteres für Schalter und Rolltreppen und das dritte, die eigentliche Busstation mit 10 Plattformen.

Sowohl von innen als auch von aussen stellt der Bau ein Netz von Dreiecken oder scheinbarem Fachwerk dar.

Die Decke setzt sich aus 14 Tetraädern zusammen, deren Seitenfronten dreieckförmige Fachwerke sind. Diese Elemente stützen sich in der Mitte des Gebäudes auf eine Reihe von gelenkig gelagerten Säulen und aussen auf die Mauern der Fassaden.

Obwohl alle Elemente, die die Struktur bilden, sehr robust sind, wirkt das Ganze doch von grosser Schlankheit. Es wurde alles an Ort und Stelle betoniert. Der Beton blieb sichtbar. 